\title{
Optimized Integration of Renewable Energy Technologies into Jordan's Power Plant Portfolio
}

\section{INCOSOL 2012,}

22/23.10.2012, Amman, Jordan

Tobias Fichter

Department of System Analysis and Technology Assessment German Aerospace Center (DLR), Stuttgart

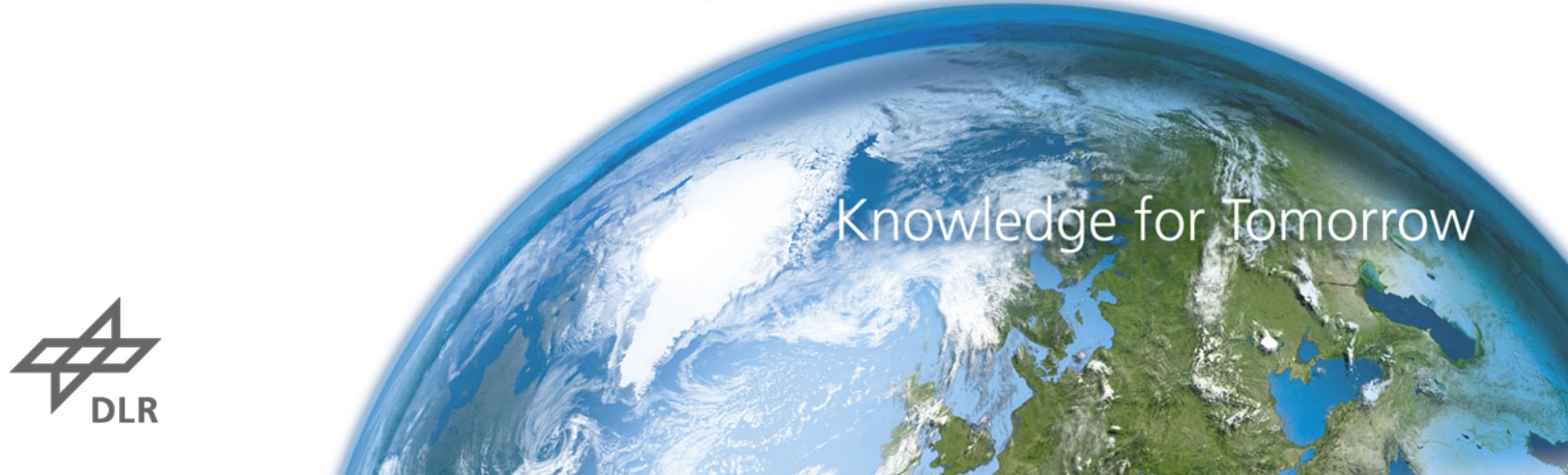




\section{Content}

1. Jordan's situation

2. Most important RE technologies for Jordan

3. Methodology

4. Results of the Jordan case study

5. Conclusion 


\section{Jordan's situation}

Strong increasing electricity demand

- Experienced growth rates of the past will continue (ca. 8\% p.a.)

- High demand of additional firm power generation capacity

- Relatively cheap N.G. from Egypt by far not enough to meet future electricity demand
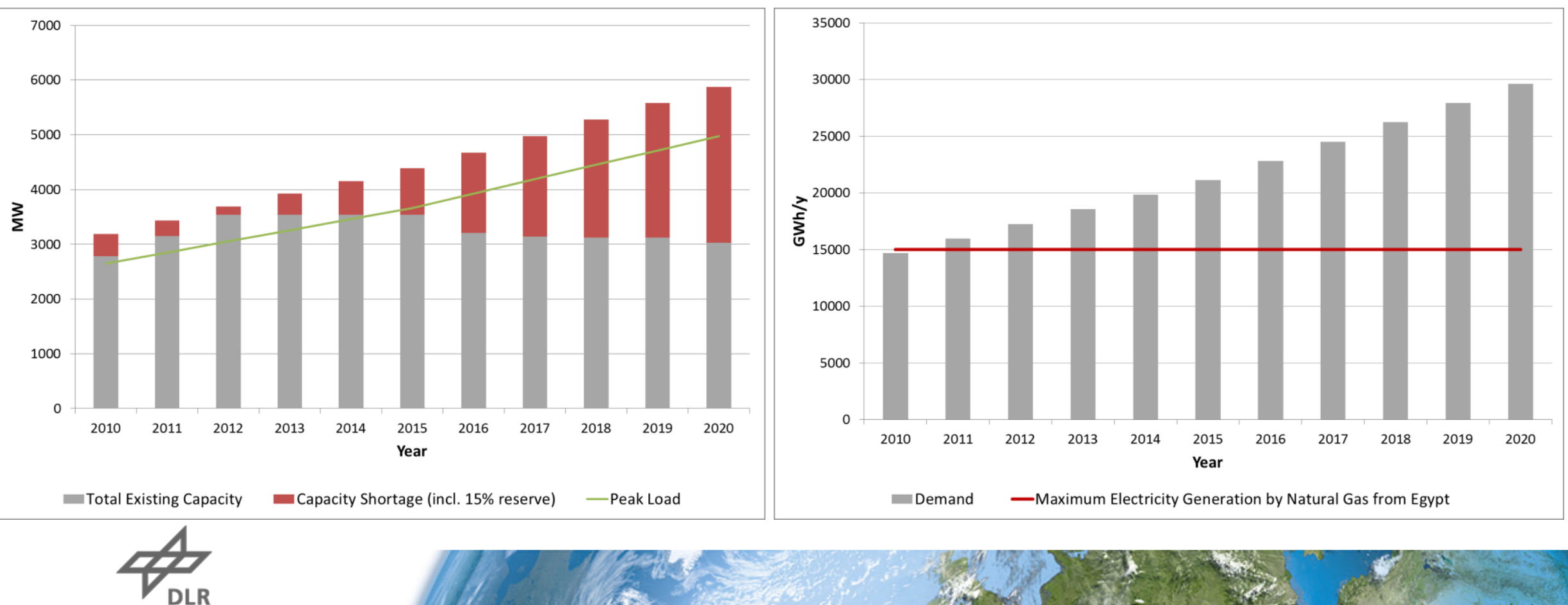


\section{Jordan's situation}

\section{Fossil fuel import dependency}

- Jordan depends highly on fossil fuel imports (ca. 96\%)

- Strong historical and prospected future fossil fuel price escalation

$\rightarrow$ Significant increase of power generation costs

\section{Existing Power Plant Capacity, 2012}

percent of total

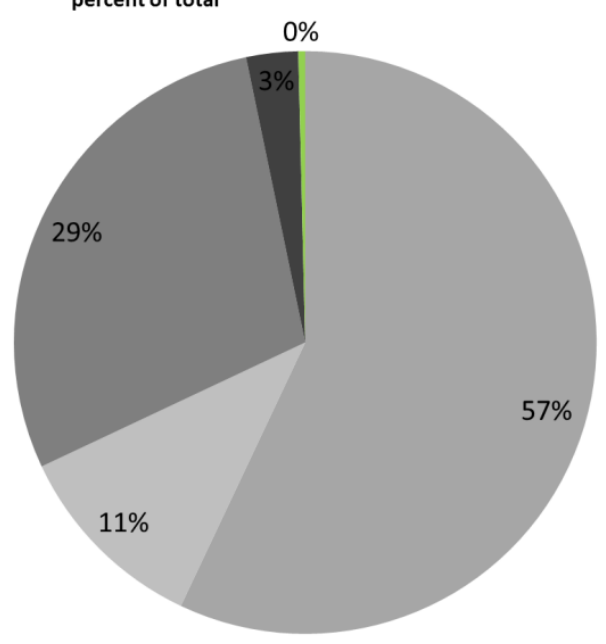

CCGT units

OCGT units

- Steam turbine units

- Inustrial sector

RES-E

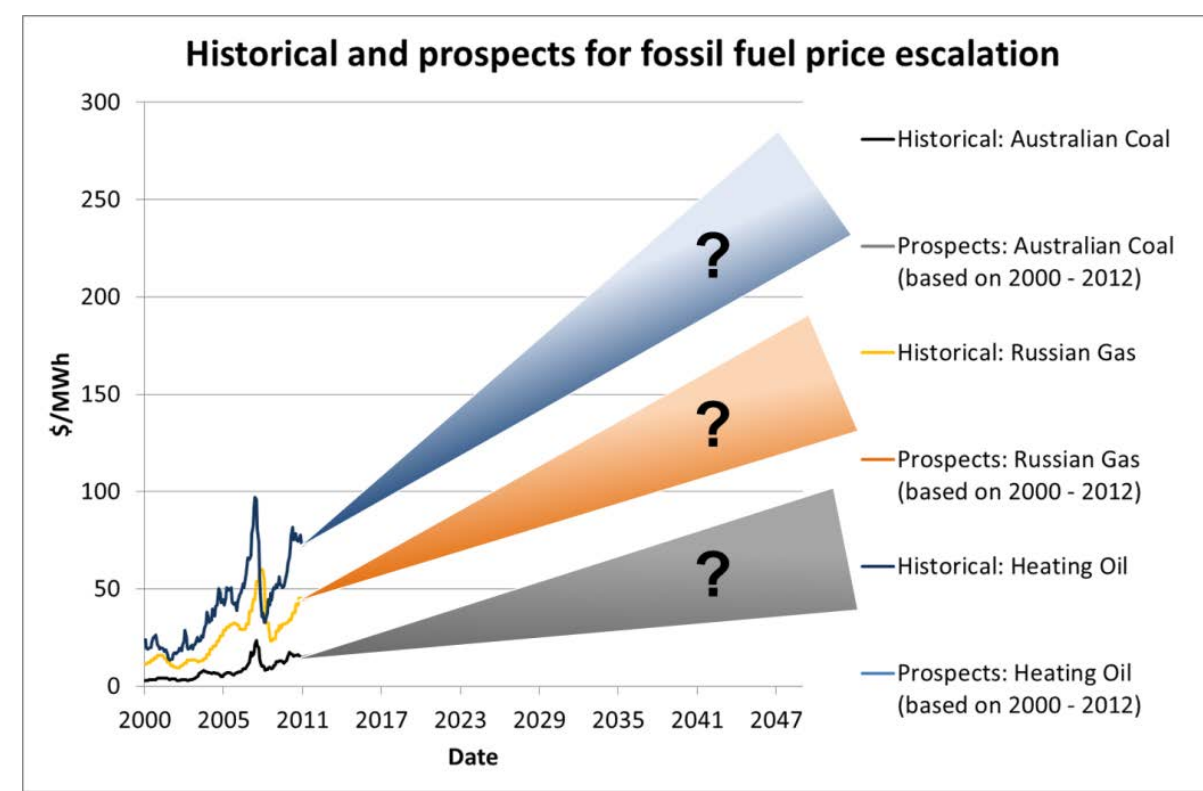




\section{Jordan's situation}

The challenge

1. Keep up with increasing electricity demand

2. Increase independency from fossil fuel imports

3. Provide electricity at reasonable prices in the future 
- Demand 2050: ca. 53 TWh/y

\section{Jordan's situation}

Outstanding RE potential
- Solar potential: ca. $6000 \mathrm{TWh} / \mathrm{y}$

- Large number of sites with outstanding solar and wind resources allowing RE power generation at very low costs

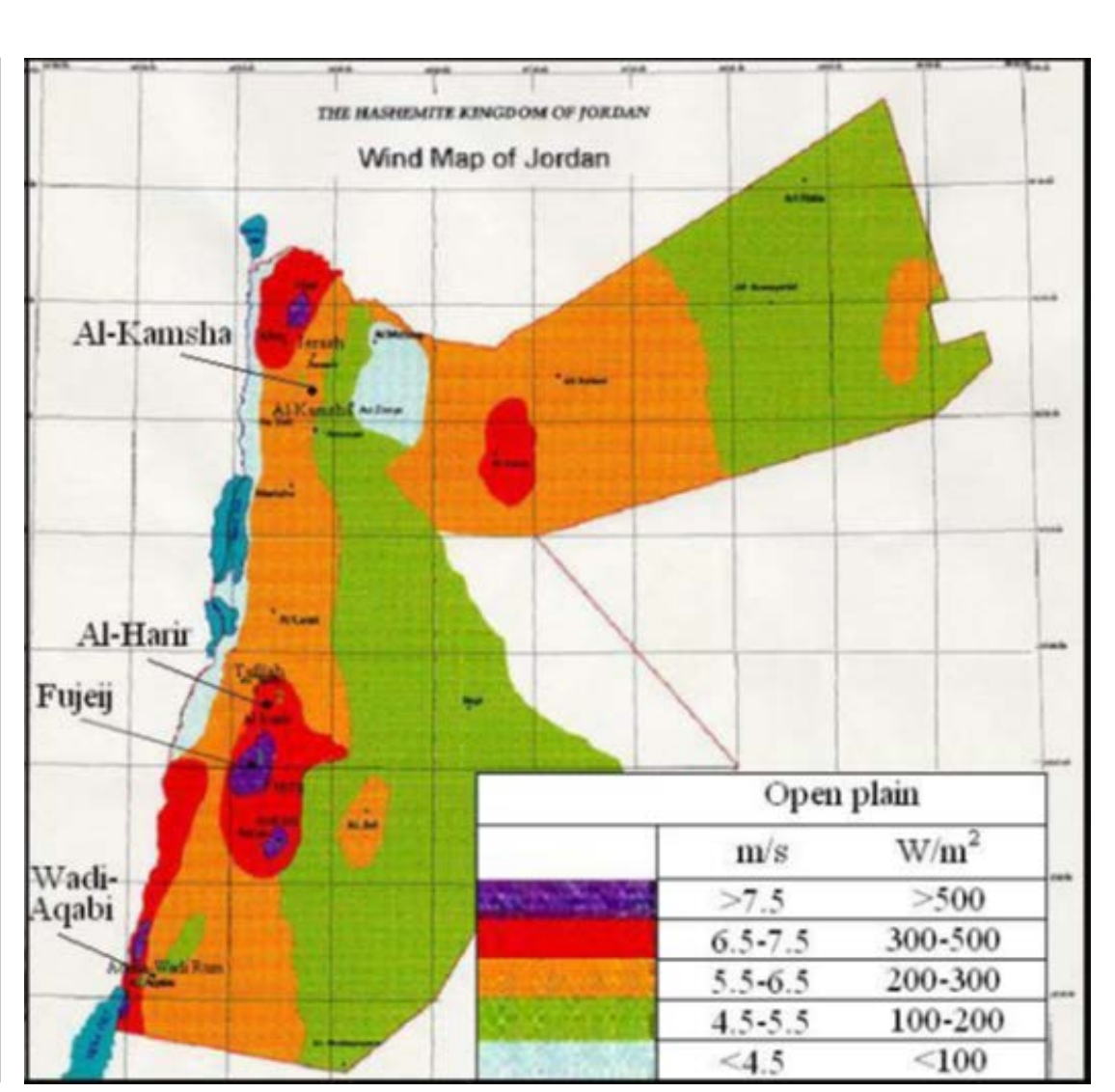

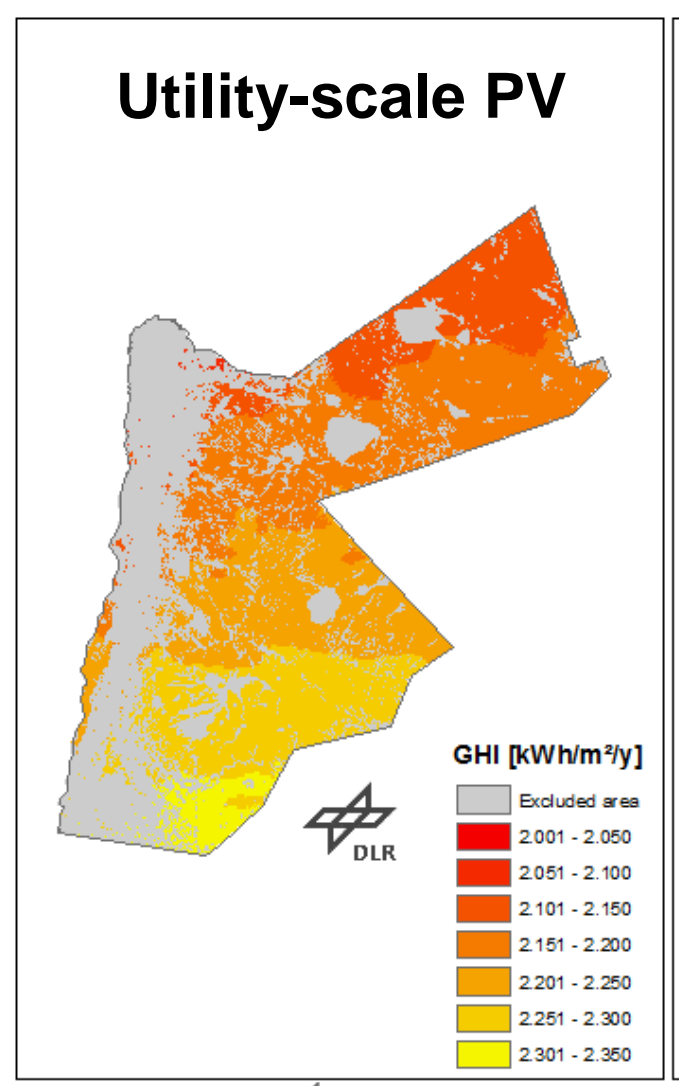
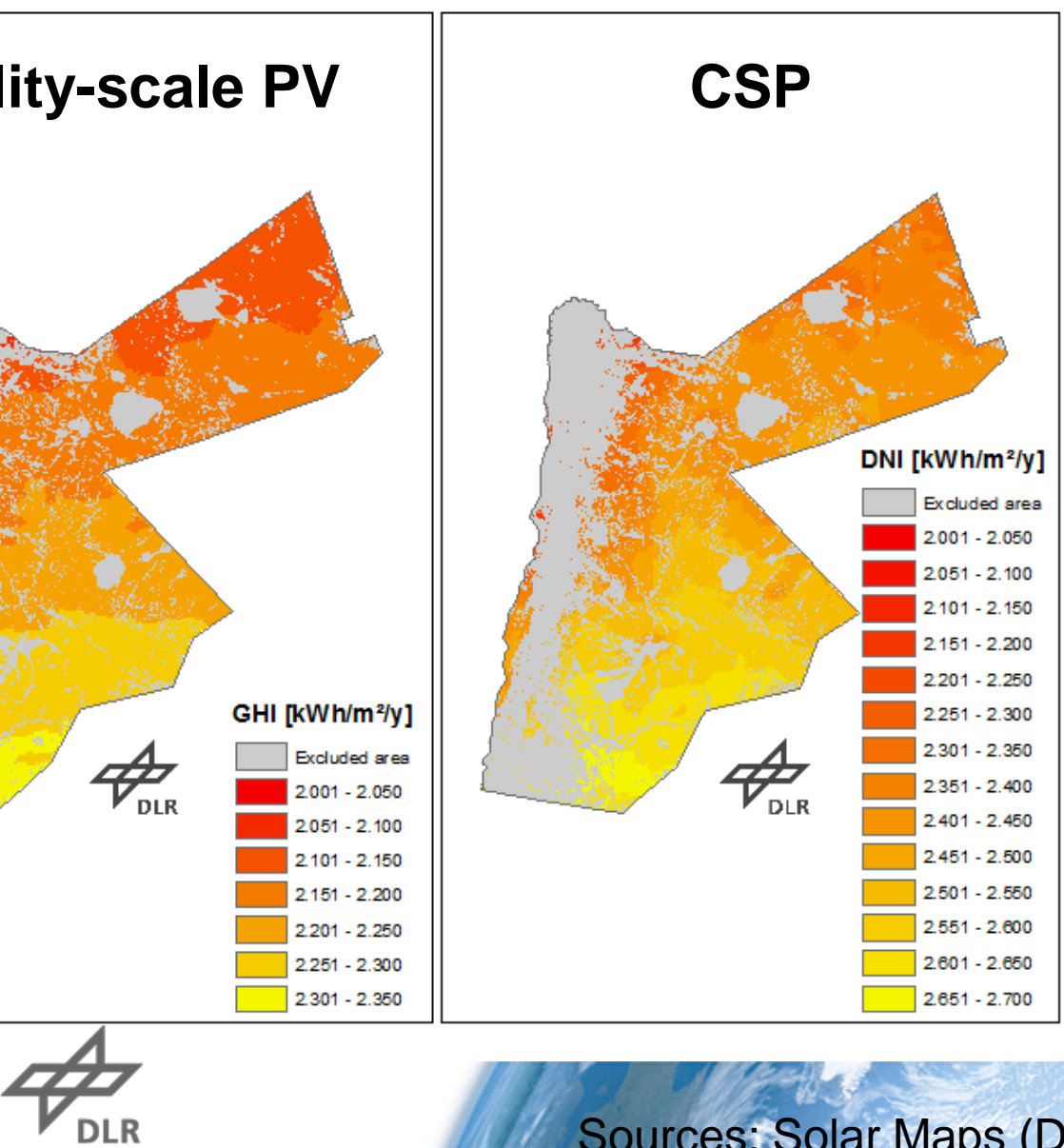

Sources: Solar Maps (DLR), Wind Map (MEMR) 


\section{Comparison of most import RE technologies for Jordan}

\begin{tabular}{|c|c|c|c|c|}
\hline & & Utility-scale PV & Onshore wind & CSP \\
\hline Global installed capacity & GW & $70 \mathrm{GW}$ & $239 \mathrm{GW}$ & $2 \mathrm{GW}$ \\
\hline Specific investment costs & $€ / k W$ & $1500-1700$ & $1200-1600$ & $2900-11000^{*}$ \\
\hline Typical plant size & MW & $0.2-100$ & $1.5-3.5$ & $20-500$ \\
\hline Typical electricity costs (MENA) & $€ / k W h$ & $0.08-0.15$ & $0.06-0.11$ & $0.14-0.22$ \\
\hline Quality of power generation & & \multicolumn{2}{|c|}{$\begin{array}{l}\text { - Fluctuating } \\
\text { - Non-dispatchable } \\
\text { - No firm capacity }\end{array}$} & $\begin{array}{l}\text { - Dispatchable } \\
\text { - Firm \& flexible } \\
\text { - System services }\end{array}$ \\
\hline
\end{tabular}

We are not looking for the cheapest single technology but we are looking for the cheapest mix of conventional and RE technologies in order to meet the future electricity demand. 


\section{Concentrating Solar Power Plants Principle of a parabolic trough CSP plant}
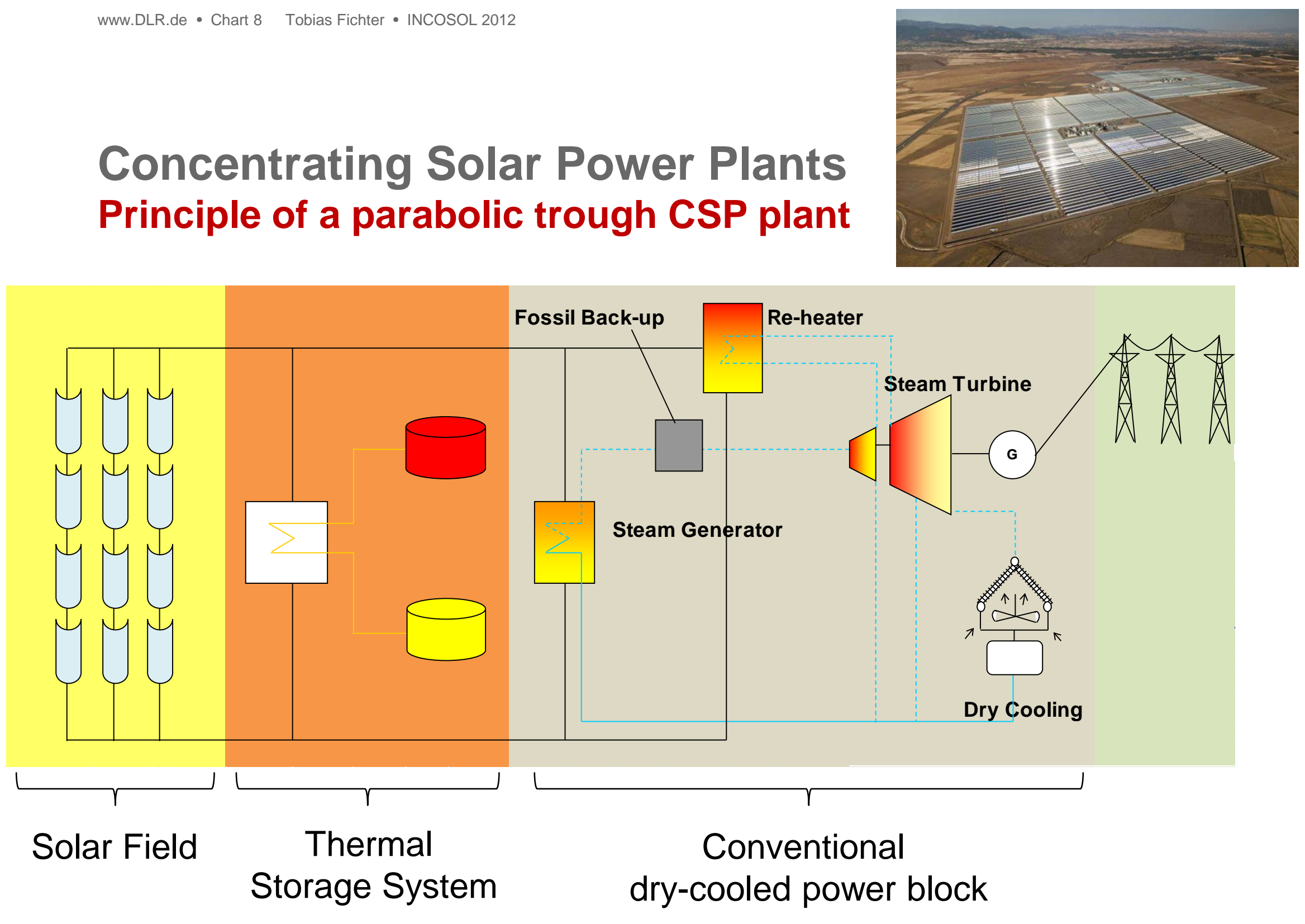


\section{Concentrating Solar Power (CSP)}

Characteristics

Investment Costs

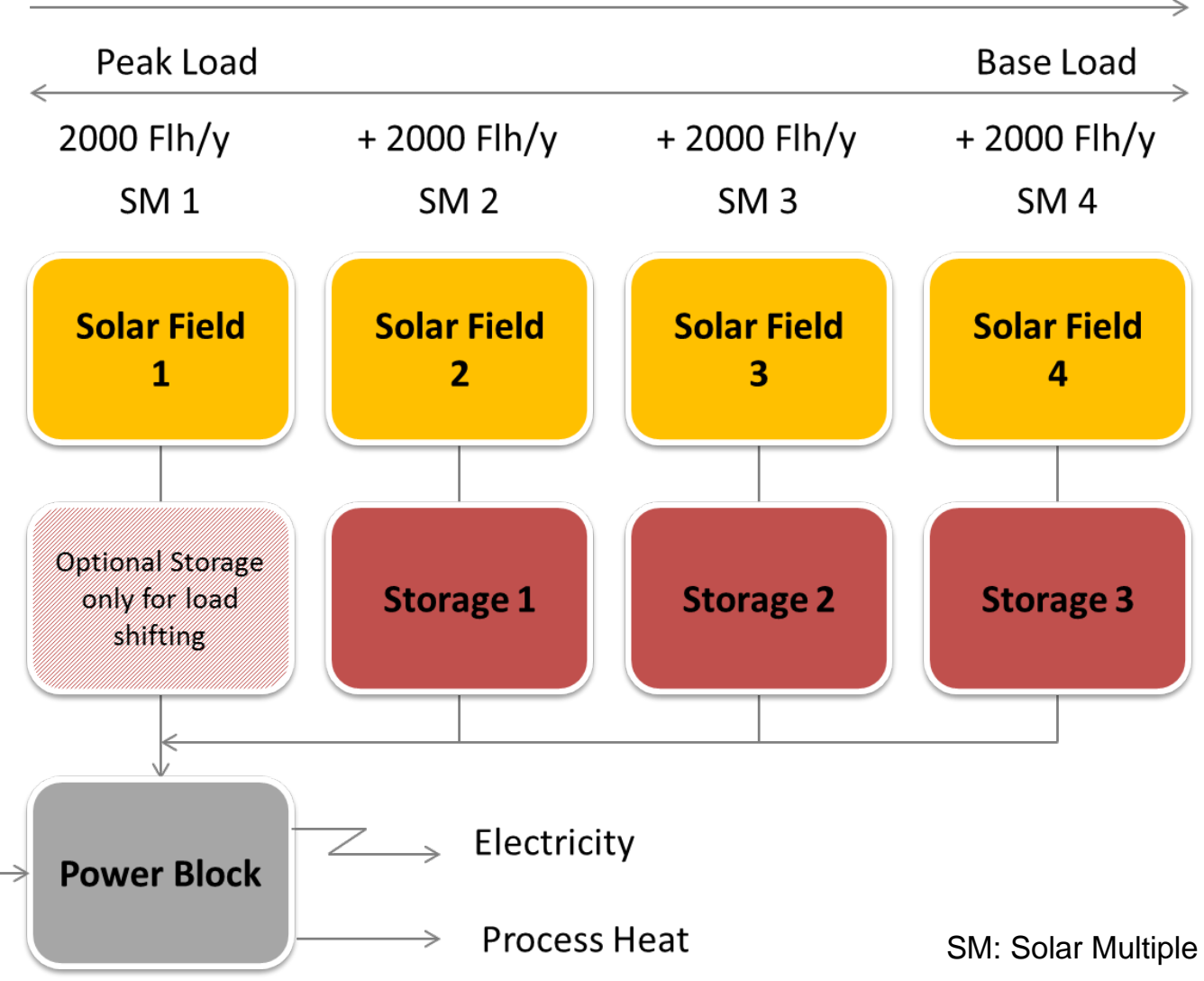

\section{Qualities of CSP Plants:}

- Operating as peak, medium or base load power plant

- Firm \& flexible capacity due to storage system and back-up boiler

- Power generation on demand

- System services

- Combined generation of process heat for industry, cooling, desalination, etc.

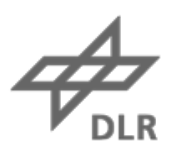




\section{Methodology for an optimized integration of RE technologies into existing power plant portfolios}

- Emphasis on cost optimized short-term integration of RE technologies and maintenance of security of electricity supply

- Results for decision support for electricity authorities and utilities in MENA

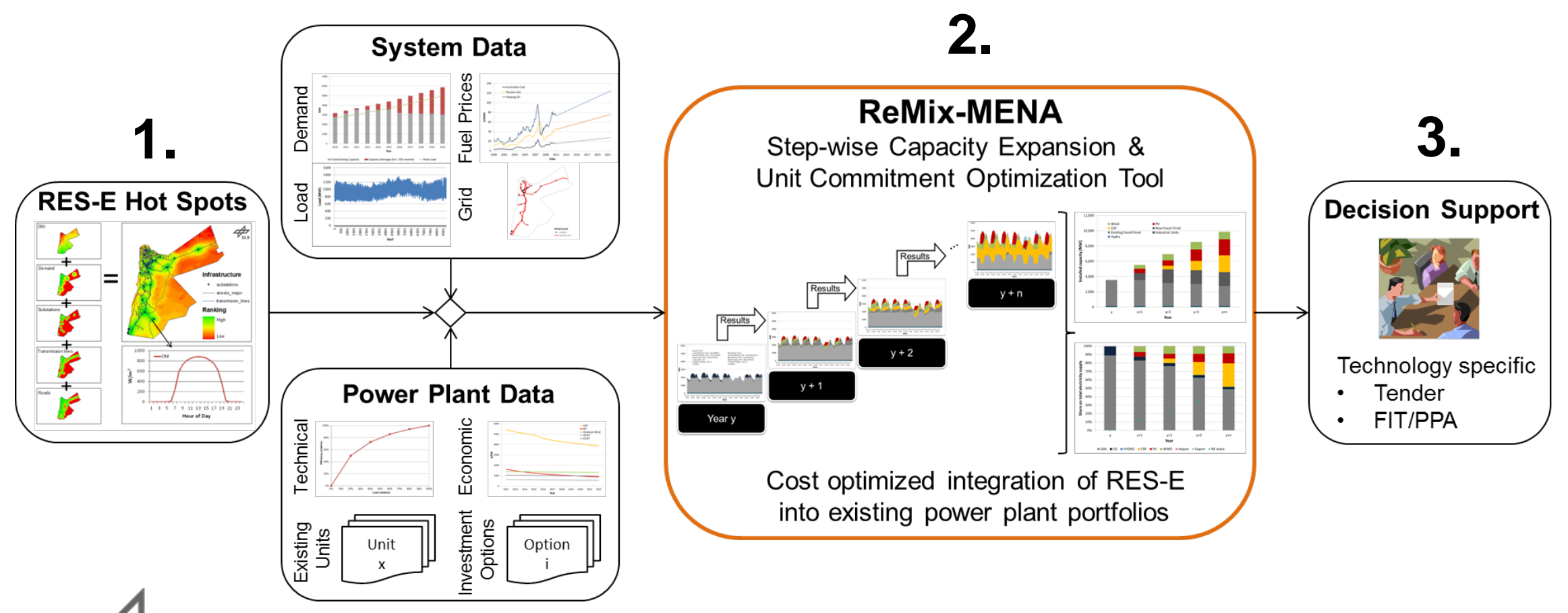




\section{Hot spots for utility-scale PV and CSP plants in Jordan} General methodology \& results
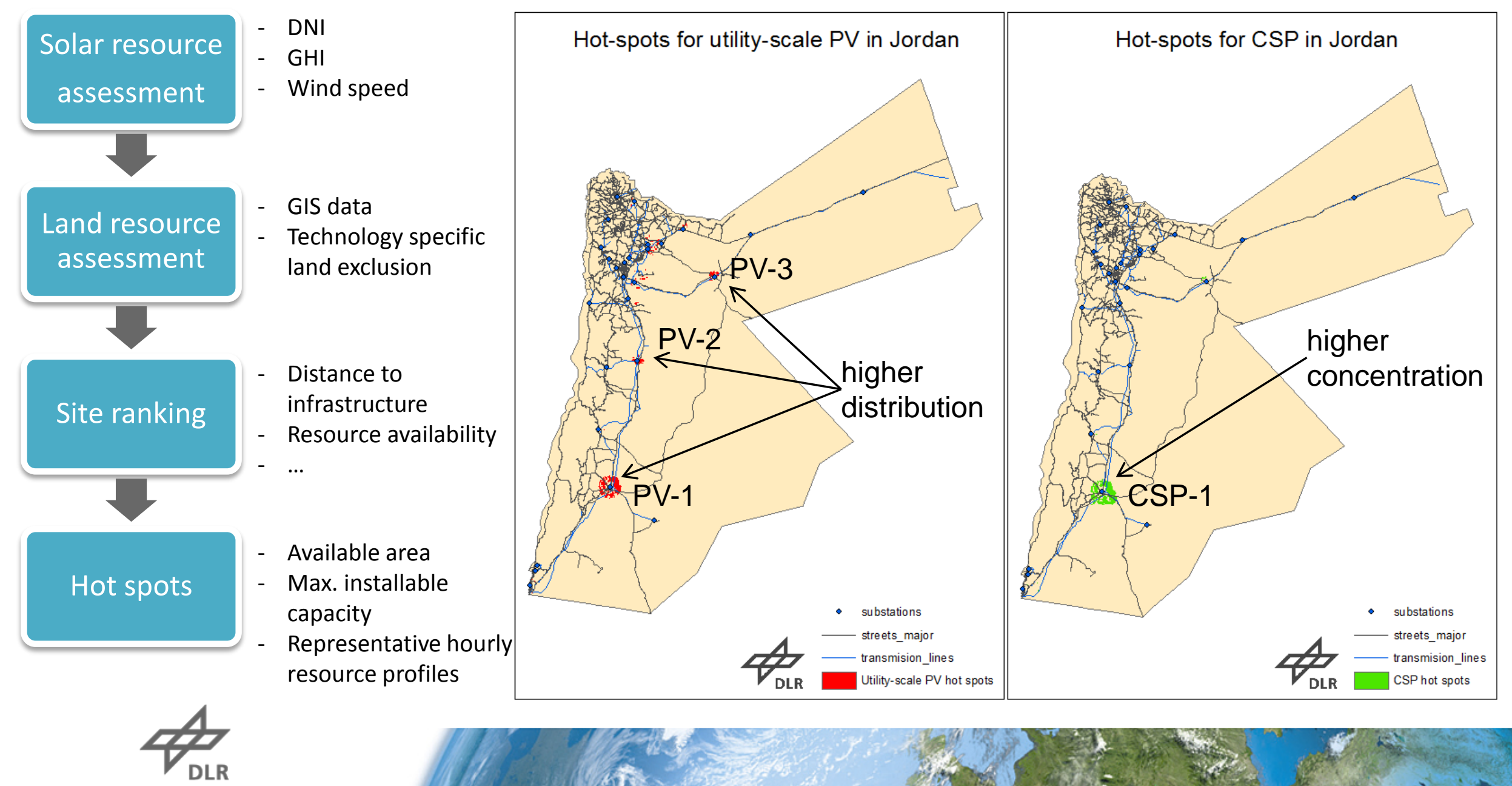


\section{Methodology for hot spot identification (site ranking)} Example CSP
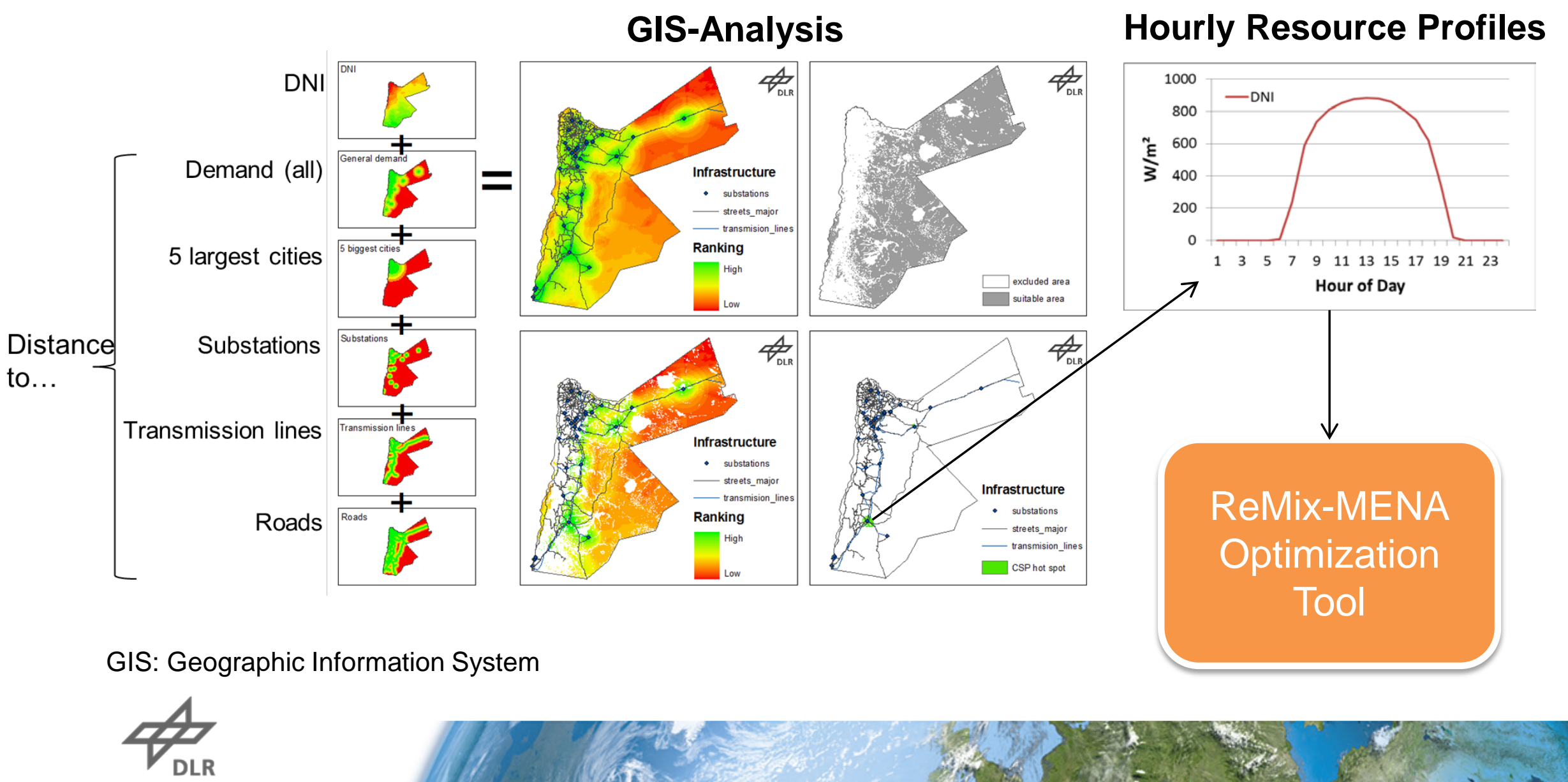


\section{ReMix-MENA:}

Optimization tool for cost efficient integration of RE technologies in MENA countries

- Model for step-wise capacity expansion, replacement and unit commitment optimization (minimization of total system costs)

- Algorithm ensures that RE technologies are only integrated when their utilization contributes to lower total power generation costs

- Starting from present power plant portfolio of the investigated country

- Detailed hourly modeling of technical and economical restrictions and dynamics of each single conventional and RE power generation unit

- Optimization of CSP configuration (solar field and storage size)

- Taking into account all necessary system restrictions (firm capacity requirements, spinning reserve, tertiary reserve, etc.) 


\section{Jordan case study} Main assumptions

- Time-frame 2012 - 2022, optimization time-steps: 2012, 2015, 2017, 2020, 2022

- Starting from Jordan's existing power plant portfolio in 2012

- Future availability of N.G. from Egypt is restricted to the amount of today

- Additional fossil fuels have to be bought at market prices

- Fossil fuel price escalation of 5\% p.a.

- Investment options: CCGT, OCGT, PV, onshore wind power and CSP units

- Investments in oil shale and nuclear power plants are excluded due to water constraints, security, economic and environmental reasons as well as very long construction times 


\section{Jordan case study Results I}

- PV, onshore wind power and CSP are already today competitive in certain load segments

- Until 2017, PV and onshore wind replacing expensive fossil fuels (LFO/Diesel)

- CSP latest from 2017 on main choice for highly required firm and flexible power capacity
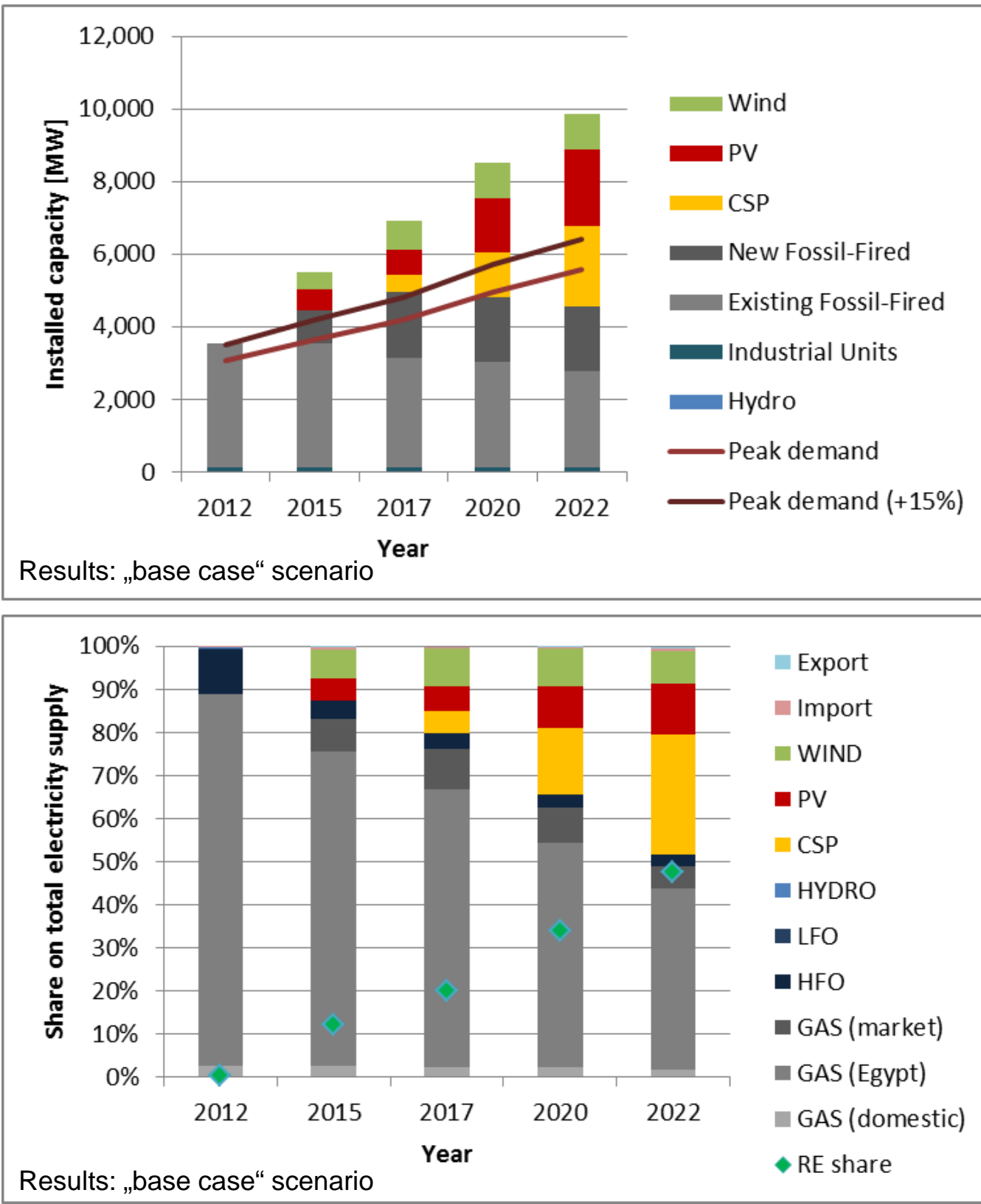


\section{Jordan case study Results II}

- PV and wind power as cheap "fossil fuel saver"

- CSP competitive in the peak and upper-mid merit sector in the short-term

- CSP providing strongly required firm and flexible generation capacity

- First CSP plants with rather small solar field and storage (SM 1.4, Storage: 5 Flh)

- In the medium-term CSP competitive with mid-merit and base load sector. SM and storage capacity is increased (up to SM 2.3, Storage 12 Flh)

- CSP in long-term as back-bone of electricity supply

Export

- Utility-scale PV - HS 1-3

CSP units - HS 1

- New CCGT units - GAS (market)

- Existing OCGT Units - LFO (market)

Existing CCGT Units - GAS (Egypt)

- Hydro Units
Import

- Onshore Wind - HS 1-4

- New OCGT units - GAS (market)

- Existing ST units - HFO (market)

- Existing OCGT units - GAS (domestic)

Industrial Units
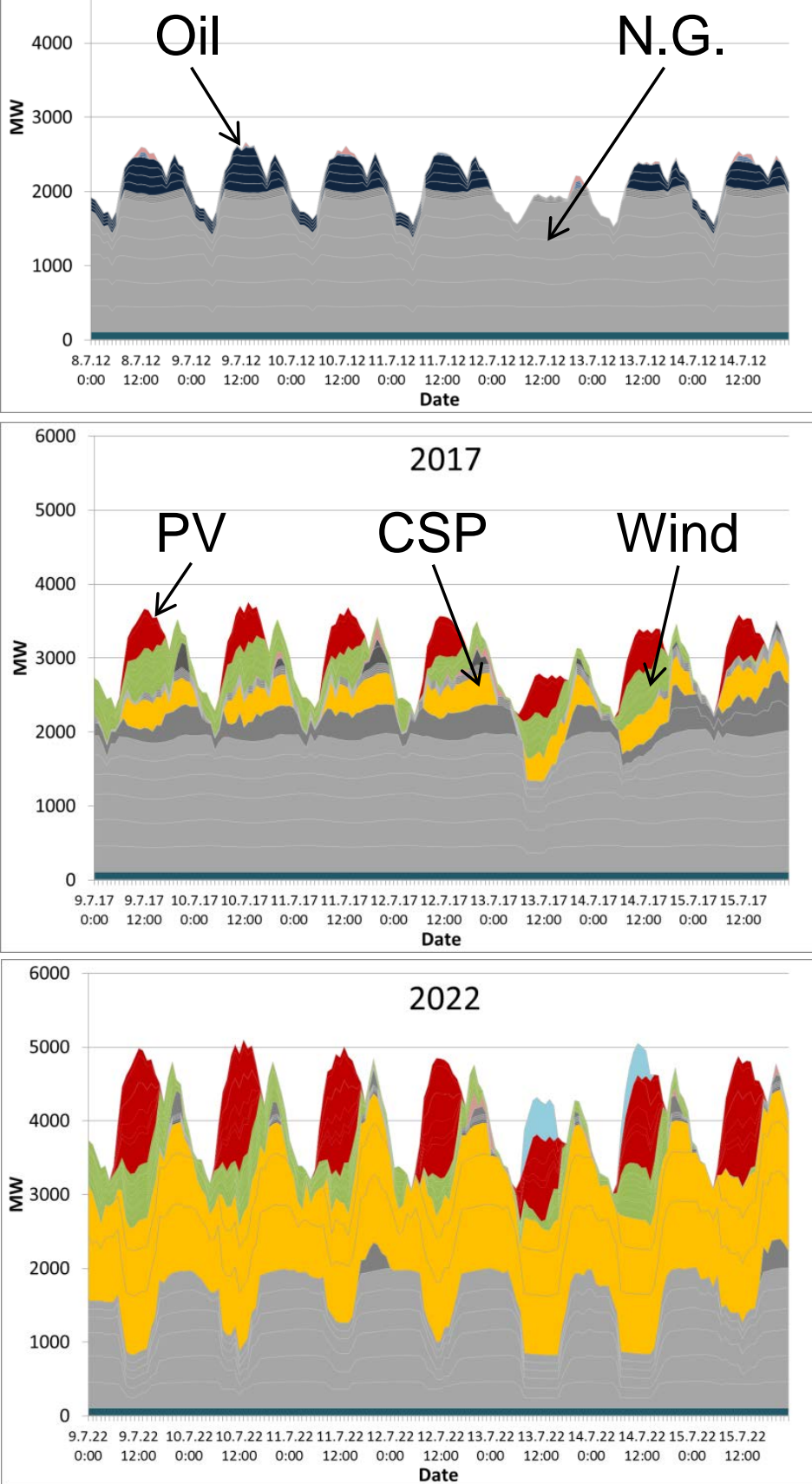


\section{Jordan case study Results III}

- Different scenarios have been investigated in addition to the "base case"

- Capacity mix of the "base case" scenario is the least cost option for Jordan to meet future electricity demand

- Large-scale integration of RE technologies will absorb significantly future electricity generation costs

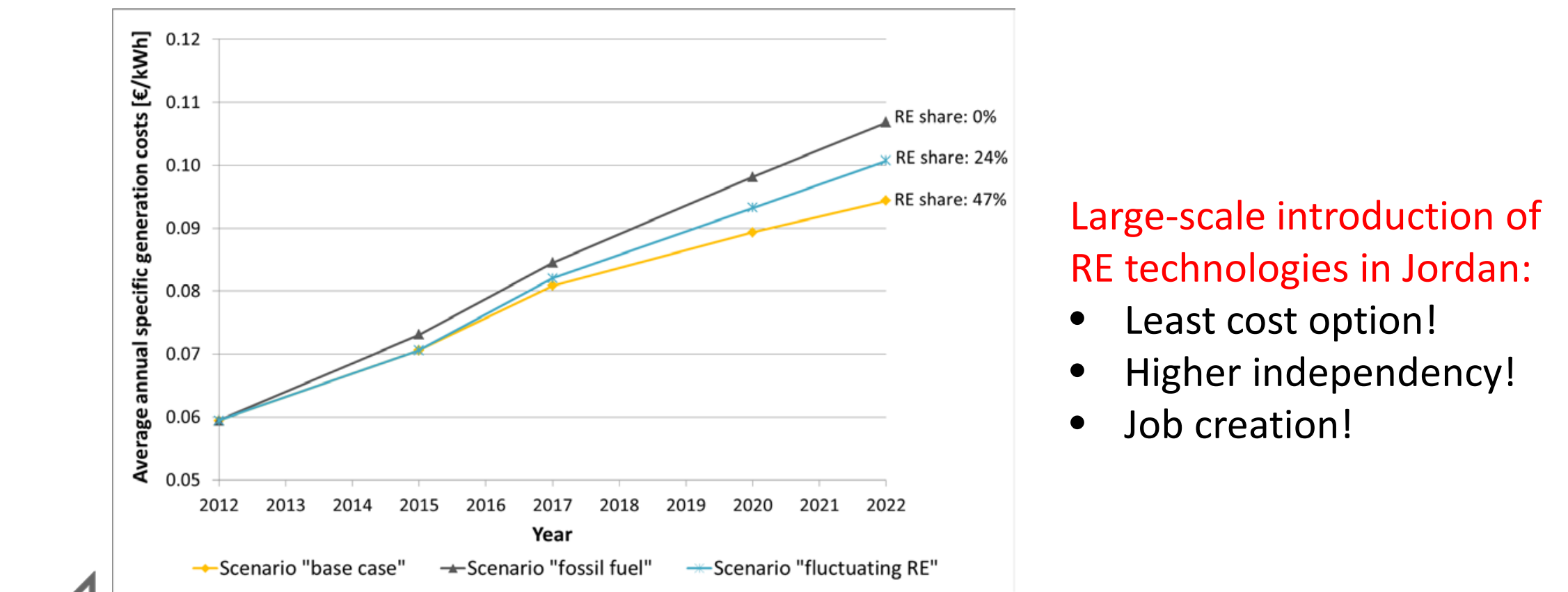




\section{Conclusions I}

\section{Jordan' situation}

- Strong increasing electricity sector

- High need for additional firm and flexible power capacity

- High dependency on fossil fuel imports

- High potential of solar and wind resources allowing low cost generation

- Very limited pump-storage potential and limited biomass potential

- CSP only large-scale alternative for firm and flexible power in Jordan except of fossil-fired power plants 


\section{Conclusions II \\ Results from ReMix-MENA}

- Today, PV, onshore wind and CSP are competitive in certain niche markets

- The large-scale integration of RE technologies and a well balanced mix of $\mathrm{RE}$ and conventional technologies is the least cost option for Jordan to keep up with the increasing electricity demand

- PV and wind power units can be used as low cost "fossil fuel saver"

- CSP most competitive technology for strongly required firm and flexible power in MENA

- CSP as backbone of electricity supply in the long-term allowing high shares of power generation by RE technologies

- The large-scale integration of RE technologies has significant economic advantages

- However, an appropriate market-framework has to be implemented 


\section{Setting an appropriate market framework}

\section{Suggestions}

- recognize the need for large $R E$ investment (RE investment replaces fuel consumption for decades!)

- Implement technology specific tenders in order to get the most suitable mix of conventional and RE power generation technologies

- reduce capital cost and thereby generation costs of RE technologies by increasing project ratings towards AAA (re-insured PPA, e.g. "World Bank Guarantee Program")

- provide transparent, long-term stable regulatory and policy framework to trigger real $\mathrm{RE}$ markets

- start immediately in order to save money! 


\section{Thank you for your attention!}

Tobias Fichter

German Aerospace Center (DLR)

Institute of Technical Thermodynamics, Stuttgart

Department of System Analysis and Technology Assessment

tobias.fichter@dlr.de

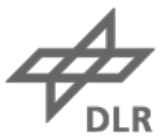




\section{Back-Up Slides}




\section{Investments in new power generation capacity Exemplary example}

- CSP plants representing firm and flexible power generation capacity with fixed generation costs

- Conventional power plants representing firm and flexible generation capacity without fixed generation costs

- CSP competitive today when performance over entire lifetime is taken into account
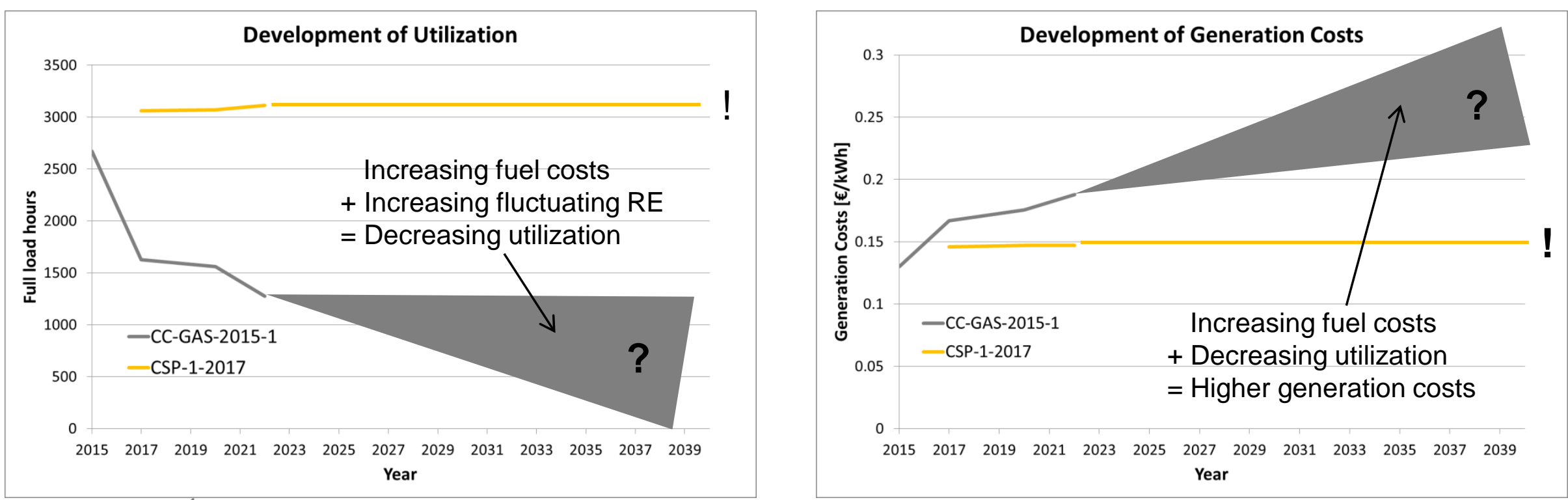

Figures: Results for 2015-2022 from ReMix-MENA ("base case"'scenario), 2023 - 2040 qualitative illustration 